\title{
ENSAIO ACELERADO DE LABORATÓRIO DE CHAPAS OSB DE SERINGUEIRA SUBMETIDAS AO ATAQUE DE FUNGOS APODRECEDORES
}

\section{ACCELERATED LABORATORY TEST OF RUBBERWOOD ORIENTED STRANDBOARD EXPOSED TO WOOD DECAY FUNGI}

\author{
Esmeralda Yoshico Arakaki Okino ${ }^{1}$ Marcus Vinícius da Silva Alves ${ }^{2}$ Divino Eterno Teixeira ${ }^{3}$ \\ Mário Rabelo de Souza ${ }^{4}$ Marcos Antonio Eduardo Santana ${ }^{5}$ Cláudio Henrique Soares Del Menezzi ${ }^{6}$
}

\section{RESUMO}

O objetivo do trabalho foi avaliar a resistência natural de chapas de partículas orientadas (OSB) confeccionadas com partículas de Hevea brasiliensis Müll.Arg. aderidas com as resinas sintéticas uréiaformaldeído (UF) e fenol-formaldeído (FF), a $5 \%$ e $8 \%$, expostas a fungos xilófagos em condições de laboratório. No ensaio acelerado de laboratório as amostras foram submetidas ao ataque dos fungos de podridão-parda Gloeophyllum trabeum (Pers. : Fr.) Murril, Coniophora puteana (Schumach. : Fr.) P. Karst., Meruliporia incrassata (Berk. \& M.A. Curtis) Murrill e também aos fungos de podridão-branca, Fomes annosus (Fr. : Fr.) Cooke, Trametes versicolor (L. : Fr.) Pilát, Ganoderma applanatum (Pers.) Pat., Bjerkandera fumosa (Pers. : Fr.) P. Karst. e Phanerochaete chrysosporium Burds. Dentre os fungos de podridão-parda, o Gloeophyllum trabeum causou a maior perda de massa nos corpos-de-prova testados. Dentre os fungos de podridão-branca, Trametes versicolor, Phanerochaete chrysosporium e Ganoderma applanatum causaram as maiores perdas de massa, sem diferença significativa para o Gloeophyllum trabeum. A resina $\mathrm{FF}$ proporcionou maior resistência à ação dos fungos nas chapas $\mathrm{OSB}$, o mesmo fato ocorrendo com o maior teor de resina. O fungo Coniophora puteana foi altamente sensível ao tipo de resina aplicado, enquanto o Bjerkandera fumosa demonstrou reduzida ação nas concentrações mais altas de ambas as resinas. $\mathrm{O}$ fungo Fomes annosus apresentou degradação insignificante para todos os tratamentos de chapas OSB.

Palavras-chave: durabilidade natural; fungos xilófagos; Hevea brasiliensis; OSB.

\section{ABSTRACT}

This work aimed to evaluate the natural durability of oriented strandboards (OSB) manufactured with strands of Hevea brasiliensis Müll.Arg. bonded with 5\% and $8 \%$ of urea-formaldehyde (UF) and phenol-formadehyde (FF) resins, exposed to xilophagous fungi under laboratory conditions. In accelerated laboratory test decay, samples of OSB were exposed to the following fungi: the brown-rot fungi Gloeophyllum trabeum (Pers. ex Fries) Murr., Coniophora puteana (Schumach. : Fr.)P. Karst., Meruliporia incrassata (Berk. \& M.A. Curtis) Murrill as well as the white-rot fungi Fomes annosus (Fr. : Fr.) Cooke, Trametes versicolor (L. : Fr.) Pilát, Ganoderma applanatum (Pers.) Pat., Bjerkandera fumosa (Pers. : Fr.) P. Karst. and Phanerochaete chrysosporium Burds. Among the brown-rot fungi, the Gloeophyllum trabeum was the most aggressive, showing the highest loss of mass. Trametes versicolor and Ganoderma applanatum confirmed the preference for broadleave species. All oriented strandboards at lower UF resin contents were more degraded by Phanerochaete chrysosporium, Trametes versicolor, Ganoderma applanatum, Merulia incrassata, Coniophora puteana and Gloeophyllum trabeum, with high rate of loss of mass. Coniophora puteana showed small loss of mass when FF resin was applied. Bjerkandera fumosa showed low loss of mass only at higher resin content. Oriented strandboards exposed to Coniophora puteana showed

1. Engenheira Química, MSc., Analista Ambiental do Laboratório de Produtos Florestais - LPF, Av. L-4 Norte SCEN, trecho 2, lote 4, bloco D, CEP 70818-900, Brasília (DF). esmeralda.okino@florestal.gov.br

2. Engenheiro Florestal, Ph.D., Gerente Executivo de Planejamento do Serviço Florestal Brasileiro - LPF, Av. L-4 Norte-SCEN, trecho 2, lote 4, bloco H, CEP 70818-900, Brasília (DF). marcus.alves@florestal.gov.br

3. Engenheiro Florestal, Ph.D., Analista ambiental do Laboratório de Produtos Florestais - LPF, Av. L-4 Norte SCEN, trecho 2, lote 4, bloco A, CEP 70818-900, Brasília (DF). divino.teixeira@florestal.gov.br

4. Físico, Ph.D., Analista ambiental do Laboratório de Produtos Florestais - LPF, Av. L-4 Norte - SCEN, trecho 2, lote 4, bloco da engenharia, CEP 70818-900, Brasília (DF). mario.souza@florestal.gov.br

5. Químico, Ph.D., Analista ambiental do Laboratório de Produtos Florestais - LPF, Av. L-4 Norte - SCEN, trecho 2, lote 4, bloco D, CEP 70818-900, Brasília (DF). marcos.santana@florestal.gov.br

6. Engenheiro Florestal, Dr., Professor Adjunto do Departamento de Engenharia Florestal, Universidade de Brasília, Caixa Postal 04357, CEP 70919-970, Brasília (DF). cmenezzi@unb.br

Recebido para publicação em 6/02/2008 e aceito em 14/08/2009. 
insignificant OSB degradation.

Keywords: natural durability; xilophagous fungi; Hevea brasiliensis; OSB.

\section{INTRODUÇÃO}

Em vista do crescente consumo de madeira reflorestada para painéis e produtos "engenheirados" em geral, faz-se necessária à melhoria da qualidade desses materiais, e o consequente aumento da vida útil.

Eaton e Hale (1993) afirmaram que a durabilidade da madeira depende das vias de acesso para os organismos se fixarem e de sua composição química. $\mathrm{O}$ acesso à penetração de fungo depende da natureza, distribuição e tamanho dos elementos celulares ou anatômicos da madeira, sendo os elementos axiais o principal meio, seguido pelos elementos radiais e tangenciais. Além desses elementos, há as barreiras naturais à penetração dos fungos, como as tiloses, pontuações, gomas e resinas. Os principais fatores químicos que influenciam a durabilidade natural da madeira são os próprios polímeros da parede celular e os extrativos presentes na forma de terpenos, e polifenóis condensados como quinonas, lignanas, taninos e estilbenos.

A madeira de seringueira (Hevea brasiliensis Müll.Arg.), mesmo sendo altamente susceptível ao ataque de organismos xilófagos, tem sido estudada por vários pesquisadores. Jusoh e Kamdem (2001), ao testarem a madeira de seringueira com 30 anos de idade, obtiveram $61,8 \%$ de perda de massa para o fungo Trametes versicolor (sinôn. Coriolus versicolor) e $61,4 \%$ para o fungo Gloeophyllum trabeum, tendo classificado a madeira como não resistente. Hong et al. (1994) afirmaram que o alto teor de carboidratos (açúcares e amido) armazenados nas células de parênquima determina a alta susceptibilidade ao apodrecimento da madeira de seringueira, fato esse corroborado por Azizol e Rahim (1989). Yamamoto e Hong (1994) apresentaram dados para quatro fungos apodrecedores, dentre eles o Coriolus versicolor, que resultou em uma perda de massa de $42,5 \%$ e a consequente classificação da madeira como não resistente. Já Sujan et al. (1980) apresentaram os seguintes valores de perdas de massa para os fungos Lenzites palisotii (25,9\%), Ganoderma applanatum (21,0\%), Trametes corrugata (11,2\%), Polyporus zonalis $(9,7 \%)$, Lentinus blepharodes (7,7\%), Schizophyllum commune (5,3\%) e Fomes senex (3,3\%).

Ainda são escassos os artigos que abordam a resistência a fungos apodrecedores de chapas OSB. Os dados de biodeterioração são mais restritos à madeira sólida e aos painéis convencionais do tipo chapas aglomeradas e compensadas. Okino et al. (2002) apresentaram os resultados de ensaio acelerado de laboratório para os fungos Gloeophyllum trabeum e Trametes versicolor em madeira sólida de seis clones de seringueira, chapas aglomerada e chapas de cimento-madeira confeccionadas com a madeira de seringueira. Tanto a madeira quanto as chapas aglomeradas foram classificadas como moderadamente resistentes enquanto as chapas de cimento-madeira não sofreram ataque. Okino et al. (2003) também testaram painéis "flakeboards" usando quatro clones de seringueira, os quais foram classificados como moderadamente resistentes quando expostos aos fungos Gloeophyllum trabeum e Trametes versicolor, resistentes ao Ganoderma applanatum e altamente resistentes ao Neolentinus lepideus (sinon Lentinus lepideus).

O objetivo do trabalho foi avaliar a resistência natural de chapas de partículas orientadas (OSB) confeccionadas com partículas de Hevea brasiliensis Müll Arg. $(\mathrm{H})$ aderidas com resinas sintéticas uréiaformaldeído (UF) e fenol-formaldeído (FF), a 5 e $8 \%$, expostas a fungos xilófagos em condições de laboratório.

\section{MATERIAL E MÉTODO}

\section{Fungos empregados}

Utilizaram-se os fungos de podridão-branca e os de podridão-parda relacionados na Tabela 1, todos pertencentes à coleção do Laboratório de Produtos Florestais do Serviço Florestal Brasileiro (LPF/SFB). A codificação dos tratamentos e dos fungos será abordada conforme descritos. 
TABELA 1: Codificações e fungos usados no ensaio de apodrecimento em chapas OSB de seringueira.

TABLE 1: Code used and fungi tested in laboratory bioassay for OSB rubberwood.

\begin{tabular}{l|c|c}
\hline \multicolumn{1}{c}{ Descrição das chapas OSB de seringueira (H) e repetições } & Resina (\%) & Tipo resina \\
\hline H5UF - 04 chapas & 5 & UF \\
H8UF - 04 chapas & 8 & UF \\
H5FF - 04 chapas & 5 & FF \\
H8FF - 04 chapas & 8 & FF \\
\hline Códigos - Fungos de podridões ensaiados & Parda & Branca \\
\hline GT - Gloeophyllum trabeum (Pers. : Fr.) Murril & & $\sqrt{ }$ \\
CP - Coniophora puteana (Schumach. : Fr.) P. Karst. & & $\sqrt{ }$ \\
MI - Meruliporia incrassata (Berk. \& M.A. Curtis) Murrill & $\sqrt{ }$ \\
FA - Fomes annosus (Fr. : Fr.) Cooke [sinôn. Heterobasidion annosum & $\sqrt{ }$ \\
(Fr. : Fr.) Bref.] & $\sqrt{ }$ \\
TV - Trametes versicolor (L. : Fr.) Pilát & $\sqrt{ }$ \\
GA - Ganoderma applanatum (Pers.) Pat. & $\sqrt{ }$ \\
BF - Bjerkandera fumosa (Pers. : Fr.) P. Karst. & $\sqrt{ }$ \\
PC - Phanerochaete chrysosporium Burds. & & \\
\hline
\end{tabular}

\section{Ensaio acelerado de laboratório e obtenção de dados}

A metodologia de ensaio acelerado de laboratório foi baseada na norma ASTM D 2017-05 (2005) e Okino et al. (2007) com pequenas modificações (ENCINAS; DANIEL, 1997; OKINO et al. 2009). A preparação do meio de cultura líquido consistiu na adição de cinco inóculos de $5 \mathrm{~mm}$ de diâmetro em erlenmeyer contendo $200 \mathrm{~mL}$ de meio malte líquido. O erlenmeyer foi mantido sob aeração por $24 \mathrm{~h}$ e depois transferidos para a incubadora por 30 dias à temperatura de $(26,7 \pm 1)^{\circ} \mathrm{C}$ e umidade relativa do ar de $(70 \pm$ 4)\%. Ao final desse período, a massa micelial com os esporos foi homogeneizada e $3 \mathrm{~mL}$ dessa suspensão foi adicionada em cada frasco de vidro de $150 \mathrm{~mL}$, que continha a terra corrigida e a placa suporte, previamente esterilizada. Após essa operação, os frascos retornaram à incubadora, à temperatura de $(26,7 \pm 1)^{\circ} \mathrm{C} \mathrm{e}$ umidade relativa do ar de $(70 \pm 4) \%$, até que a placa suporte estivesse totalmente encoberta por micélio.

Corpos-de-prova condicionados a $(50 \pm 2)^{\circ} \mathrm{C}$, até massa constante, foram pesados, esterilizados e introduzidos assepticamente nos frascos de vidro preparados e inoculados. Após as 12 semanas de exposição aos fungos xilófagos, removeu-se o excesso de massa micelial da superfície dos corpos-de-prova, que foram estabilizados nas condições iniciais de $(50 \pm 2)^{\circ} \mathrm{C}$. A resistência das chapas OSB foi avaliada por meio da porcentagem da perda de massa, conforme norma ASTM D 2017-05 (2005), dada pela Tabela 2 abaixo.

TABELA 2: Classificação da resistência natural, segundo a norma ASTM D 2017-05.

TABLE 2: Decay resistance expressed as either weight loss or residual weight according to ASTM D 201705.

\begin{tabular}{c|c|l}
\hline Perda de peso (\%) & Peso residual (\%) & Classe de resistência indicada para um fungo específico \\
\hline 0 a 10 & 90 a 100 & Altamente resistente - AR \\
11 a 24 & 76 a 89 & Resistente - R \\
25 a 44 & 56 a 75 & Moderadamente resistente - MR \\
45 ou mais & Menos de 56 & Pouco ou não resistente - NR \\
\hline
\end{tabular}

Delineamento experimental

Utilizaram-se as chapas confeccionadas por Okino et al. (2006) com "strands" médio de $0,70 \mathrm{x} 20 \mathrm{x}$ 70 mm de dimensão e uma mistura proporcional de dois clones, Tjir 16 e RRIM 600, de seringueira (Hevea brasiliensis Müll.Arg.) para o ensaio acelerado de laboratório. Chapas de três camadas, na proporção de face $/$ miolo de $50: 50$ e densidade nominal de $0,75 \mathrm{~g} / \mathrm{cm}^{3}$, coladas com $5 \%$ e com $8 \%$ de resinas ureiaformaldeído (UF) e fenol-formaldeído(FF), perfazendo quatro tratamentos com quatro repetições cada, totalizando 16 chapas. O clone Tjir 16 com 30 anos de idade estava em fase de abate e o clone RRIM 600 com 17 anos estava no auge da exploração de látex. Ambos os clones eram cultivados no estado de São Paulo.

O delineamento experimental quanto ao ensaio acelerado de laboratório constou da combinação de 
duas porcentagens de resina ( $5 \%$ e $8 \%$ ), dois tipos de resinas (UF e FF) e oito fungos (GT, CP, MI, FA, TV, GA, BJ e PC) totalizando 32 tratamentos. Foram utilizados três corpos-de-prova de cada uma das quatro chapas de repetições, perfazendo 12 corpos-de-prova por tratamento. Utilizaram-se placas suportes de alburno de Cecropia sp. para os fungos de podridão-branca e de Pinus sp. para os de podridão-parda.

Os resultados foram submetidos a uma análise de variância e os fatores que foram significativos pelo teste de $\mathrm{F}$ tiveram suas médias submetidas ao teste de Tukey $(\mathrm{p}<0,05)$. O software utilizado para a análise dos dados foi o "Statistical Program for Social Science (SPSS), versão 13.0 para "Windows".

\section{RESULTADOS E DISCUSSÃO}

$\mathrm{Na}$ análise separada por teor e tipo de resina e espécie de fungo, os resultados foram os descritos nas Figuras 1, 2, e 3, em que as barras são as médias e as hastes em "T" a média $\pm 1,0$ desvio-padrão. A letra inserida em cada barra representa a separação de média em grupos pelo teste de Tukey.

\section{Efeito da porcentagem de resina das chapas OSB no ataque de fungos}

Os valores da Figura 1 mostram que os teores de resinas foram fatores significativos ao nível de 1\% de significância, em que a maior proporção apresentou uma menor perda de massa, ou seja, a proporção de $5 \%$ apresentou perda de massa média de $19,26 \%$ e a de $8 \%$ de $13,37 \%$.

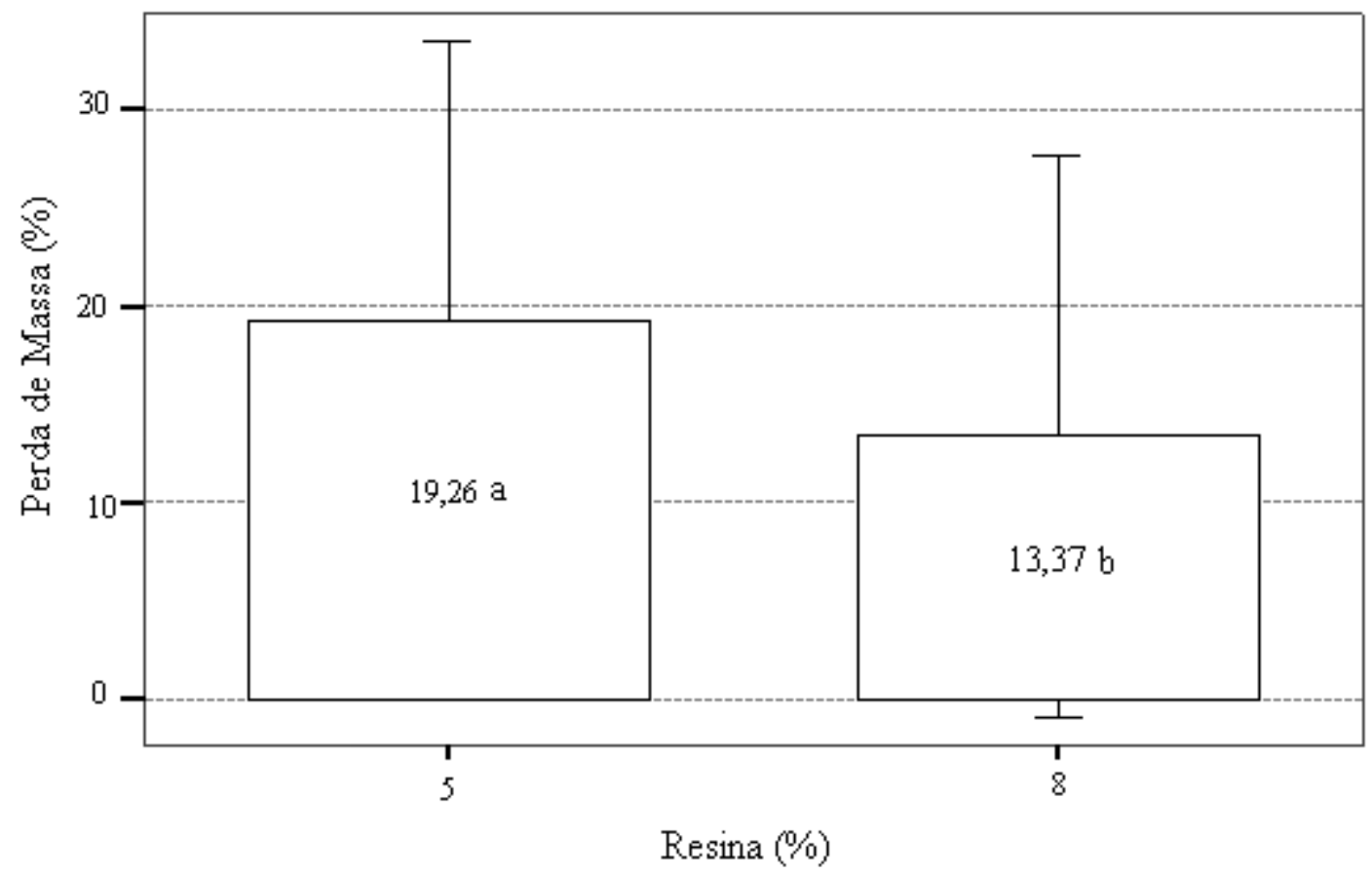

FIGURA 1: Efeito da porcentagem de resina nas chapas OSB de seringueira expostas aos fungos apodrecedores.

FIGURE 1: Effect of the resin content in rubberwood OSB exposed to decay fungi.

\section{Efeito do tipo de resina nas chapas OSB no ataque de fungos}

Pelos dados da Figura 2, percebe-se que o tipo de resina foi estatisticamente significativo ao nível de $1 \%$ de significância, em que a resina $\mathrm{FF}$ apresentou o menor valor de perda de massa médio $(8,95 \%)$. Na verdade, as resinas $\mathrm{FF}$ interagem menos com a água ou umidade do ar, tornando-as mais resistentes à absorção e ao inchamento do que as ureicas, impedindo que as amostras atinjam o teor de umidade ideal ao crescimento e atuação dos fungos xilófagos. Por outro lado, Kartal e Green III (2003), afirmaram que as resinas ureicas não são resistentes à água, em que o inchamento das amostras proporciona maior acesso aos fungos às fibras da madeira. No entanto, Walther et al. (2007) relataram que a degradação por fungos de chapas coladas com resina fenólicas é tão severa quanto aquelas coladas com resina ureica. 


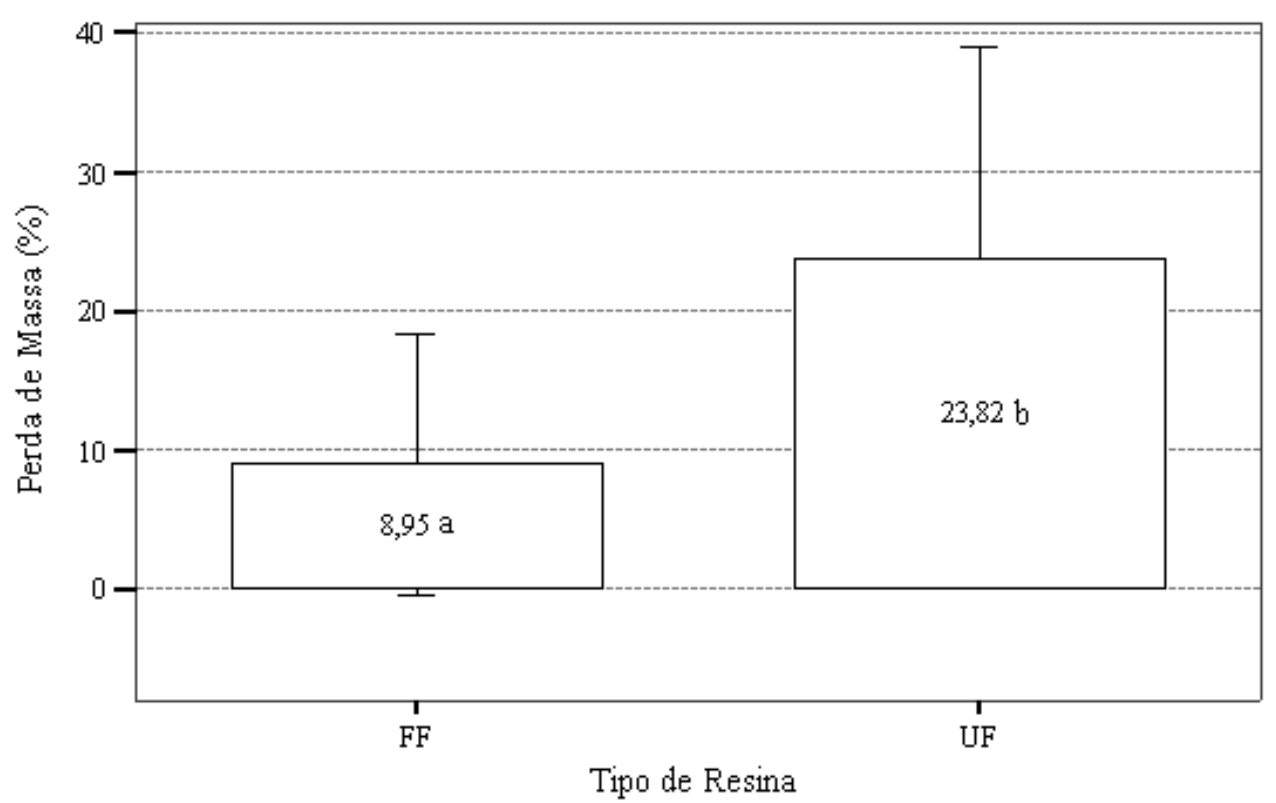

FIGURA 2: Efeito do tipo de resina em chapas OSB de seringueira quando exposto a diferentes tipos de fungos apodrecedores.

FIGURE 2: Effect of the type of resin in rubberwood OSB exposed to different types of decay fungi.

\section{Efeito dos fungos nas chapas OSB}

Os fungos exibiram comportamentos diferenciados ao nível de 1\% de significância, apresentando três grupos homogêneos (Figura 3). O grupo de fungos que ocasionou a maior perda de massa média foi o Trametes versicolor, Phanerochaete chrysosporium, Ganoderma applanatum e Gloeophyllum trabeum, sem diferença significativa entre eles. O que ocasionou a menor perda foi o fungo Fomes annosus. Vale ressaltar que, apesar da maioria desses fungos serem de podridão-branca, o mais agressivo continua sendo o de podridão-parda.

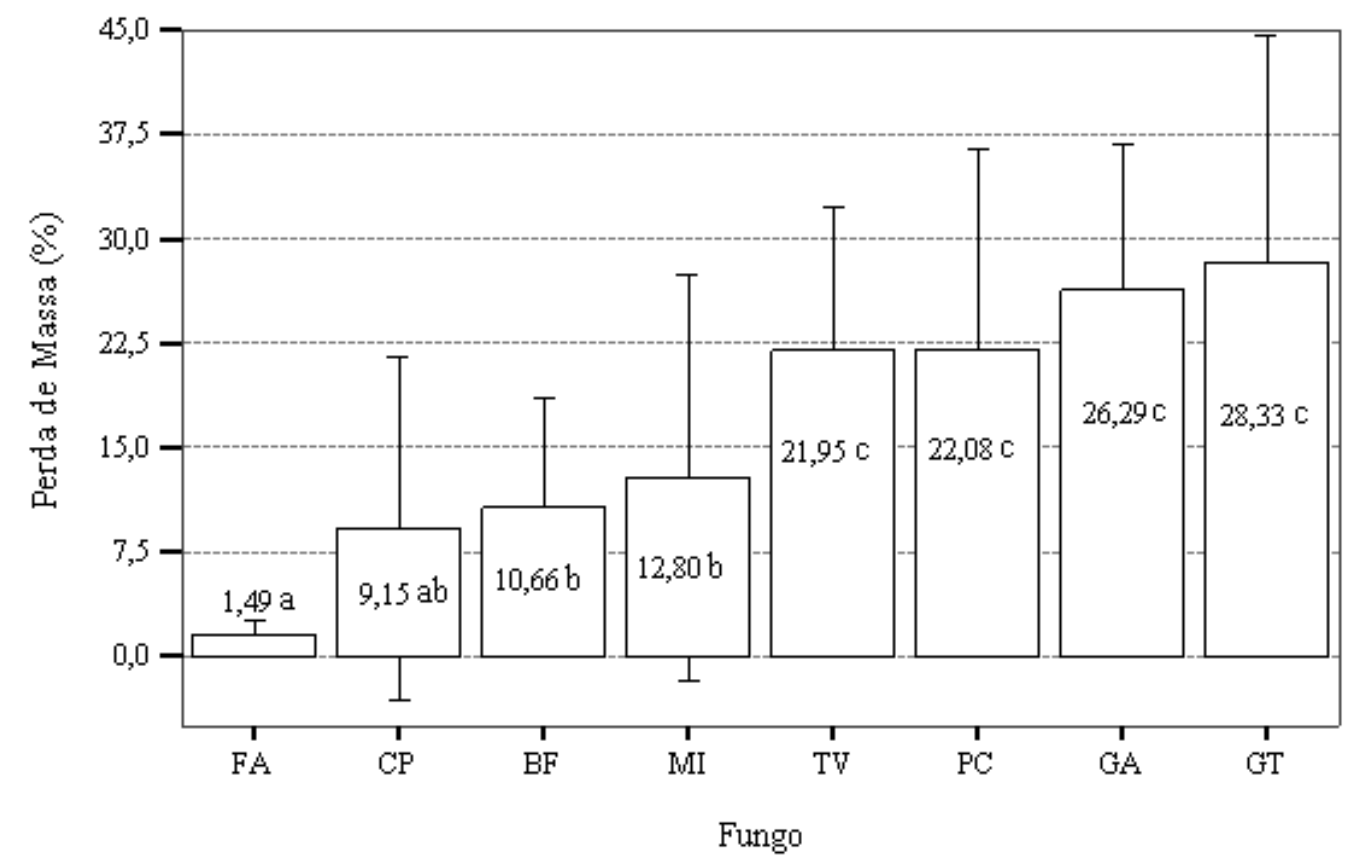

FIGURA 3: Efeito do comportamento de diferentes espécies de fungos testados em chapas OSB de seringueira submetidas ao ensaio acelerado de laboratório.

FIGURE 3: Effect of several fungi species in OSB exposed to accelerated laboratory assay. 


\section{Efeito das interações}

A análise de variância das interações entre as porcentagens e tipos de resinas e os tipos de fungos, além desses fatores isolados estão apresentados na Tabela 3 e foram estatisticamente significativos a $1 \%$ de significância. Isso quer dizer que a perda de massa só é explicada por esse conjunto de fatores inter-relacionados.

TABELA 3: Análise de variância das interações entre porcentagens de resinas, tipos de resinas e fungos testados em chapas OSB de seringueira no ensaio acelerado de laboratório.

TABLE 3: Analysis of variance between-subjects effects in rubberwood OSB exposed to accelerated laboratory test.

\begin{tabular}{l|c|c|c|c|}
\hline \multicolumn{5}{|c|}{ Variável dependente: Perda de Massa (\%) } \\
\hline Fonte de variação & $\begin{array}{c}\text { Soma dos } \\
\text { quadrados }\end{array}$ & $\begin{array}{c}\text { Graus de } \\
\text { liberdade }\end{array}$ & $\begin{array}{c}\text { Quadrado } \\
\text { médio }\end{array}$ & F calculado \\
\hline Modelo corrigido & $55230,954(\mathrm{a})$ & 31 & 1781,644 & $60,642^{* *}$ \\
Intersecção & 83783,438 & 1 & 83783,438 & $2851,767 * *$ \\
\%Resinas & 3151,536 & 1 & 3151,536 & $107,270^{* *}$ \\
Tipos Resinas & 14231,752 & 1 & 14231,752 & $484,411^{* *}$ \\
Fungos & 21440,796 & 7 & 3062,971 & $104,255^{* *}$ \\
\%Resinas * Tipos Resinas & 694,652 & 1 & 694,652 & $23,644^{* *}$ \\
\%Resinas * Fungos & 3492,092 & 7 & 498,870 & $16,980^{* *}$ \\
Tipos Resinas * Fungos & 6711,666 & 7 & 958,809 & $32,635^{* *}$ \\
\%Resinas * Tipos Resinas * Fungos & 1614,553 & 7 & 230,650 & $7,851^{* *}$ \\
\hline
\end{tabular}

Em que: (a) $\mathrm{R}^{2}=0,875\left(\mathrm{R}^{2}\right.$ ajustado $\left.=0,860\right) .{ }^{* *}$ Significativo a $1 \%$ de probabilidade $(\mathrm{p}<0,01)$.

Os resultados da porcentagem da perda de massa de amostras de OSB de seringueira, expostas aos fungos de podridão-parda e de podridão-branca no ensaio acelerado de laboratório estão expressos na Tabela 4.

As chapas OSB com 5\% de resina UF, quando expostas ao ataque de fungos, apresentaram resistências variadas, sendo moderadamente resistentes (MR) aos fungos Merulia incrassata, Coniophora puteana, Gloeophyllum trabeum, Phanerochaete chrysosporium, Trametes versicolor e Ganoderma applanatum, resistentes (R) ao fungo Bjerkandera fumosa e altamente resistentes (AR) ao fungo Fomes annosus. Por sua vez, aquelas com $8 \%$ de resina UF foram classificadas como MR com relação aos fungos Gloeophyllum trabeum, Phanerochaete chrysosporium, Trametes versicolor e Ganoderma applanatum, R ao fungo Coniophora puteana e AR aos fungos Merulia incrassata, Fomes annosus e Bjerkandera fumosa. Esses resultados indicam que o incremento de 3\% no teor de resina UF proporcionou uma menor perda de massa, ou seja, as chapas se tornaram menos susceptíveis ao apodrecimento.

Analisando as chapas OSB com 5\% de resina FF expostas ao ataque de fungos apodrecedores, obtiveram-se as seguintes classes de resistência: MR ao fungo Phanerochaete chrysosporium, $\mathrm{R}$ aos fungos Merulia incrassata, Gloeophyllum trabeum, Bjerkandera fumosa, Trametes versicolor e Ganoderma applanatum e AR aos fungos Coniophora puteana e Fomes annosus, por outro lado, com $8 \%$ de resina FF, a nova sequência foi R ao fungo Ganoderma applanatum, AR aos fungos Merulia incrassata, Coniophora puteana, Fomes annosus, Gloeophyllum trabeum, Bjerkandera fumosa, Phanerochaete chrysosporium e Trametes versicolor. Nota-se que as chapas OSB com $8 \%$ de resina FF apresentaram um comportamento homogêneo diante da maioria dos fungos testados, ressaltando o efeito inibidor do tipo de resina.

Os resultados da Tabela 4 mostram que as chapas OSB com 5 e $8 \%$ de resina UF foram classificadas como moderadamente resistentes, quando expostas aos fungos Trametes versicolor e Gloeophyllum trabeum. Essa mesma classe de resistência foi encontrada por Okino et al. (2002) cujo valor é a média de perda de peso dos clones RRIM 600 e Tjir 16 para a madeira de seringueira. Comparativamente à madeira sólida, o fungo Trametes versicolor apresentou certa inibição diante da resina UF das chapas OSB confeccionadas.

O teor de resina FF nas chapas OSB teve uma ação inibitória contra os fungos Merulia incrassata, Gloeophyllum trabeum, Bjerkandera fumosa, Phanerochaete chrysosporium, Trametes versicolor e Ganoderma applanatum sobretudo na concentração de resina mais elevada. Esse resultado era previsível, em 
função do potencial biocida de compostos fenólicos.

TABELA 4: Perda de massa (\%) de chapas OSB de seringueira expostas aos fungos testados. TABLE 4: Loss of mass (\%) of rubberwood OSB exposed to tested fungi.

\begin{tabular}{|c|c|c|c|c|}
\hline \multirow[b]{2}{*}{ Tratamentos } & \multicolumn{4}{|c|}{ Perda de massa (\%) e classe de resistência } \\
\hline & $\begin{array}{l}\text { Merulia incrassata } \\
\text { Podridão-parda }\end{array}$ & $\begin{array}{l}\text { Classe de resistência } \\
\text { ASTM - D 2017-05 }\end{array}$ & $\begin{array}{c}\text { Coniophora puteana } \\
\text { Podridão-parda }\end{array}$ & $\begin{array}{l}\text { Classe de resistência } \\
\text { ASTM - D 2017-05 }\end{array}$ \\
\hline $\begin{array}{l}\text { H5UF } \\
\text { H5FF } \\
\text { H8UF } \\
\text { H8FF }\end{array}$ & $\begin{array}{c}34,64 \pm 13,50 \mathrm{hij} \\
22,96 \pm 12,77 \mathrm{defg} \\
7,86 \pm 4,28 \mathrm{ab} \\
2,63 \pm 0,52 \mathrm{a}\end{array}$ & $\begin{array}{c}\text { MR } \\
\text { R } \\
\text { AR } \\
\text { AR }\end{array}$ & $\begin{array}{c}29,74 \pm 12,15 \mathrm{fghi} \\
1,25 \pm 0,95 \mathrm{a} \\
23,49 \pm 7,47 \mathrm{defg} \\
2,44 \pm 0,35 \mathrm{a}\end{array}$ & $\begin{array}{c}\text { MR } \\
\text { AR } \\
\text { R } \\
\text { AR }\end{array}$ \\
\hline Tratamentos & $\begin{array}{l}\text { Gloeophyllum trabeum } \\
\text { Podridão-parda }\end{array}$ & $\begin{array}{l}\text { Classe de resistência } \\
\text { ASTM - D 2017-05 }\end{array}$ & $\begin{array}{l}\text { Fomes annosus } \\
\text { Podridão-branca }\end{array}$ & $\begin{array}{l}\text { Classe de resistência } \\
\text { ASTM - D 2017-05 }\end{array}$ \\
\hline $\begin{array}{l}\text { H5UF } \\
\text { H5FF } \\
\text { H8UF } \\
\text { H8FF }\end{array}$ & $\begin{array}{c}39,42 \pm 9,45 \mathrm{ij} \\
15,54 \pm 5,81 \mathrm{bcd} \\
40,35 \pm 6,66 \mathrm{ij} \\
4,88 \pm 2,80 \mathrm{a} \\
\end{array}$ & $\begin{array}{l}\text { MR } \\
\text { R } \\
\text { MR } \\
\text { AR } \\
\end{array}$ & $\begin{array}{r}0,86 \pm 0,57 \mathrm{a} \\
1,01 \pm 0,37 \mathrm{a} \\
1,96 \pm 1,56 \mathrm{a} \\
2,14 \pm 0,17 \mathrm{a} \\
\end{array}$ & $\begin{array}{l}\text { AR } \\
\text { AR } \\
\text { AR } \\
\text { AR }\end{array}$ \\
\hline Madeira $^{1}$ & $30,58 \pm 1,85$ & MR & - & - \\
\hline \multirow[b]{2}{*}{ Tratamentos } & \multicolumn{4}{|c|}{ Perda de massa $(\%)$ e classe de resistência } \\
\hline & $\begin{array}{l}\text { Bjerkandera fumosa } \\
\text { Podridão-branca }\end{array}$ & $\begin{array}{l}\text { Classe de resistência } \\
\text { ASTM - D 2017-05 }\end{array}$ & $\begin{array}{l}\text { Phanerochaete } \\
\text { chrysosporium } \\
\text { Podridão-branca }\end{array}$ & $\begin{array}{l}\text { Classe de resistência } \\
\text { ASTM - D 2017-05 }\end{array}$ \\
\hline $\begin{array}{l}\text { H5UF } \\
\text { H5FF } \\
\text { H8UF } \\
\text { H8FF }\end{array}$ & $\begin{array}{c}15,70 \pm 6,30 \mathrm{bcd} \\
15,77 \pm 7,95 \mathrm{bcd} \\
9,61 \pm 6,10 \mathrm{abc} \\
2,39 \pm 0,32 \mathrm{a}\end{array}$ & $\begin{array}{l}\mathrm{R} \\
\mathrm{R} \\
\mathrm{AR} \\
\mathrm{AR}\end{array}$ & $\begin{array}{c}31,87 \pm 9,24 \text { ghij } \\
27,42 \pm 9,03 \text { efgh } \\
32,50 \pm 4,74 \text { ghij } \\
3,22 \pm 1,13 \text { a }\end{array}$ & $\begin{array}{l}\text { MR } \\
\text { MR } \\
\text { MR } \\
\text { AR }\end{array}$ \\
\hline Tratamentos & $\begin{array}{l}\text { Trametes versicolor } \\
\text { Podridão-branca }\end{array}$ & $\begin{array}{l}\text { Classe de resistência } \\
\text { ASTM - D 2017-05 }\end{array}$ & $\begin{array}{c}\text { Ganoderma applanatum } \\
\text { Podridão-branca }\end{array}$ & $\begin{array}{l}\text { Classe de resistência } \\
\text { ASTM - D 2017-05 }\end{array}$ \\
\hline $\begin{array}{l}\text { H5UF } \\
\text { H5FF } \\
\text { H8UF } \\
\text { H8FF }\end{array}$ & $\begin{array}{c}30,72 \pm 2,13 \text { ghij } \\
18,73 \pm 3,51 \text { cde } \\
29,78 \pm 2,29 \text { fghi } \\
7,97 \pm 3,19 \mathrm{ab}\end{array}$ & $\begin{array}{l}\text { MR } \\
\text { R } \\
\text { MR } \\
\text { AR }\end{array}$ & $\begin{array}{c}31,02 \pm 4,44 \text { ghij } \\
20,60 \pm 1,83 \mathrm{def} \\
39,35 \pm 11,36 \mathrm{ij} \\
17,10 \pm 5,89 \mathrm{bcd}\end{array}$ & $\begin{array}{c}\text { MR } \\
\mathrm{R} \\
\mathrm{MR} \\
\mathrm{R}\end{array}$ \\
\hline Madeira $^{1}$ & $37,70 \pm 6,68$ & MR & - & - \\
\hline
\end{tabular}

Em que: Médias seguidas por uma mesma letra, em uma coluna específica, não são estatisticamente diferentes pelo teste de Tukey a 5\% de significância. ${ }^{1}$ Extraído de Okino et al.(2002).

Os fungos Merulia incrassata, Gloeophyllum striatum e Bjerkandera fumosa responderam negativamente ao incremento da concentração das resinas UF e FF, os fungos Phanerochaete chrysosporium e Trametes versicolor ao incremento da resina fenólica, e o fungo Coniophora puteana ao incremento da resina ureica. $\mathrm{O}$ aumento no teor de resina causou a redução da perda de massa por meio da inibição da atividade fúngica.

No geral, foi constatado o efeito inibidor do teor e do tipo de resina na ação dos fungos e, consequentemente, nas classes de resistências apresentadas pelas chapas OSB. No entanto, o efeito do tipo de resina prevaleceu sobre o teor de resina.

As chapas OSB com 5\% de resina UF e FF, expostas ao fungo Merulia incrassata, apresentaram três e quatro grupos homogêneos respectivamente, inferindo-se que, nessa concentração, o tipo de resina foi estatisticamente significativo.

As chapas OSB com 5\% de resina UF e FF, expostas ao fungo Bjerkandera fumosa, apresentaram três grupos homogêneos, em que o tipo e o teor de resina não foram fatores significativos. No entanto, a $8 \%$ de resina UF e FF apresentaram três e um grupo homogêneo respectivamente, muito embora ambas tenham sido classificadas como altamente resistentes, o teste de Tukey demonstrou haver diferença significativa entre os tipos e os teores de resinas.

As chapas OSB com 5 e com $8 \%$ de resina FF, expostas ao fungo Coniophora puteana, foram classificadas como altamente resistentes, apresentando um único grupo homogêneo onde o teor de resina não foi estatisticamente significativo. Por outro lado, as chapas OSB com 5 e com $8 \%$ de resina UF foram 
classificadas como moderadamente resistentes e resistentes, respectivamente, apresentando quatro grupos homogêneos.

As chapas OSB com 5 e $8 \%$ de resina UF submetidas aos fungos apodrecedores Gloeophyllum trabeum, Phanerochaete chrysosporium, Trametes versicolor e Ganoderma applanatum apresentaram a mesma classe de durabilidade, implicando que o incremento de $3 \%$ no teor de resina UF não melhorou a classe de resistência biológica. Isto pode significar uma redução expressiva no custo operacional, caso as demais propriedades tecnológicas estejam em conformidade com o uso. Fazendo a mesma analogia com a resina FF, as classes de durabilidade das chapas OSB melhoraram quando ensaiadas com os fungos Meruliporia incrassata, Gloeophyllum trabeum, Bjerkandera fumosa, Phanerochaete chrysosporium e Trametes versicolor.

As chapas orientadas de seringueira foram bastante atacadas pelos fungos Meruliporia incrassata, Gloeophyllum trabeum, Phanerochaete chrysosporium, Trametes versicolor e Ganoderma applanatum, no ensaio acelerado, oscilando entre três classes de resistências. Esse comportamento era previsível, pois a madeira de seringueira possui um caráter próprio, apresenta alto teor de carboidratos presentes no alburno da madeira, de aproximadamente $8 \%$, na forma de grãos de amido distribuídos no interior de células parenquimáticas, comparativamente a outras espécies, que oscila entre 1 a 3\% (SANTANA; EIRAS, 1999).

Se, por um lado, o tratamento preservativo é facilitado pela estrutura anatômica porosa e pelos elementos celulares com finas paredes facilitando a movimentação da umidade e do ar, por outro, cria canais de acesso, aumenta a permeabilidade e a aeração, que associadas a essa fonte primária de nutrientes torna a madeira de seringueira altamente susceptível ao ataque de organismos xilófagos como os fungos, coleópteros e térmitas.

Dentre todos os tratamentos estudados, nenhuma chapa OSB foi classificada como não resistente diante dos oito fungos testados. Esses resultados são bastante promissores em se tratando da madeira de seringueira, que foi cientificamente retratada como susceptível aos fungos xilófagos.

$\mathrm{O}$ fungo Fomes annosus foi o único que apresentou um único grupo homogêneo para os quatro tratamentos testados, o que pode estar relacionado ao ataque específico, que é a doença da raiz em árvores (CRAIG et al., 2000). Dentre os oito fungos testados, cinco demonstraram relativa perda de massa, mas em conformidade aos valores descritos na literatura para painéis reconstituídos.

\section{CONCLUSÕES}

As chapas orientadas de madeira de seringueira foram susceptíveis a Meruliporia incrassata, Gloeophyllum trabeum, Phanerochaete chrysosporium, Trametes versicolor e Ganoderma applanatum. O Gloeophyllum trabeum apresentou a maior perda de massa média nas chapas OSB.

Os fungos de podridão-branca Phanerochaete chrysosporium, Trametes versicolor e Ganoderma applanatum apresentaram perda de massa média similar ao fungo de podridão-parda Gloeophyllum trabeum.

$\mathrm{O}$ incremento de $3 \%$ no teor de resina $\mathrm{FF}$ apresentou um efeito positivo na classe de resistência das chapas OSB, contrariamente à resina UF, que não apresentou diferença significativa.

Os fungos Meruliporia incrassata, Gloeophyllum trabeum, Bjerkandera fumosa, Phanerochaete chrysosporium, Trametes versicolor e Ganoderma applanatum apresentaram elevado potencial de degradação, especialmente nas chapas ureicas com baixa concentração.

Nenhum dos tratamentos testados, nas chapas OSB, foi classificado como não resistente, quando expostos aos oito fungos apodrecedores.

\section{AGRADECIMENTOS}

Os autores agradecem o apoio dos Técnicos Ambientalistas Ricardo P. de O. Santos; Getúlio F. de Almeida, Luiz D. Santana e Francisco L. de Araújo e o suporte administrativo de Eudes A. Pereira, J. C. Mendes, Maria A. Maranhão, Maria H. M. G. de Figueiredo e Hugulino de A. Dias.

\section{REFERÊNCIAS BIBLIOGRÁFICAS}

AMERICAN SOCIETY FOR TESTING AND MATERIALS- ASTM D 2017. Standard method of accelerated laboratory test of natural decay resistance of woods. Annual Book of ASTM Standard. Philadelphia, v. 0410. 2005. AZIZOL, A. K.; RAHIM, S. Carbohydrates in rubberwood (Hevea brasiliensis Muell Arg.) Holzforschung, Berlin, 
V. 43, n. 3, p. 173-178, Jan 1989.

CRAIG, L. S. et al. Annosus root disease of Western Conifers. Forest Service. U.S. Department of Agriculture, 2000, 9 p. (Forest Insect \& Diseases Leaflet, 172)

EATON, R. A.; HALE, M. D. C. Wood: decay, pests and protection. Cambridge: Chapman \& Hall, 1993. 541 p.

ENCINAS, O., DANIEL, G. Degradation of the gelatinous layer in aspen and rubberwood by the blue stain fungus Lasiodiplodia theobromae. IAWA Journal, Leiden, v.18, p.107-115, 1997.

HONG, L. T. et al. Durability of rubberwood. In: Hong, L. T.; Lim, S. C. (Eds.). Rubberwood processing and utilization. Kuala Lumpur: Forest research Institute of Malaysia, p.37-50, 1994.

JUSOH, I.; KAMDEM. D. P. Laboratory evaluation of natural decay resistance and efficacy of CCA-treated rubberwood (Hevea brasiliensis Muell. Arg.). Holzforschung, Berlin, v. 55, n. 3, p. 250-254, Apr. 2001.

KARTAL, S. N.; GREEN III, F. Decay and termite resistance of medium density fiberboard (MDF) made from different wood species. International Biodeterioration \& Biodegradation, Birmingham, v. 51, p. 29-35, 2003.

OKINO, E. Y. A. et al. Color variation of rubberwood clones and cypress infected by Gloeophyllum striatum and Phanerochaete chrysosporium. International Biodeterioration \& Biodegradation, Birmingham, v. 63, p. 41-45, 2009.

OKINO, E. Y. A. et al. Biodegradação de chapas de partículas orientadas de pinus, eucalipto e cipreste expostas a quatro fungos apodrecedores. Scientia Forestalis, Piracicaba, n. 74, p. 67-74, 2007.

OKINO, E. Y. A. et al. Propriedades de chapas OSB com espécies de madeira de florestas plantadas no Brasil: strands de $80 \mathrm{~mm}$ de Hevea brasiliensis. In: CONGRESSO BRASILEIRO DE INDUSTRIALIZAÇÃO DA MADEIRA PRODUTOS DE BASE FLORESTAL, 2., 2006, Curitiba. Anais...Curitiba, 2006, 10 p.

OKINO, E. Y. A. et al. Propriedades físicas, mecânicas e durabilidade natural de chapas aglomeradas de Hevea brasiliensis Müell Arg. In: CONGRESSO FLORESTAL BRASILEIRO, 8., 2003, São Paulo. Anais...São Paulo, 2003, Pôster n. 324.

OKINO, E. Y. A. et al. Durabilidade natural de madeira sólida, de chapas aglomeradas e de chapas de cimento-madeira de Hevea brasiliensis. Brasil Florestal, Brasília, v. 73, p. 39-46, 2002.

SANTANA, M. A. E.; EIRAS, K. M. M. Madeira de Hevea brasiliensis: adequação tecnológica para sua utilização. Brasília: IBAMA/DIGET, 1999. 90 p. (Relatório Interno).

SUJAN, A. et al. Some studies on fungal deterioration of rubberwood (Hevea brasiliensis). International Research Group on Wood Preservation, 1980, 9 p. (Document N. IRG/WP/2140).

WALTHER, T. et al. Strength, decay and termite resistance of oriented kenaf fiberboards. J. Wood Sci, Japan, v. 53, p. 481-486, 2007.

YAMAMOTO, K.; HONG, L. T. A laboratory method for predicting the durability of tropical hardwoods. Japan Agricultural Research Quaterly, Japan, v. 28, p. 268-275, 1994. 